\title{
ON GROUPS WITH FINITELY MANY INDECOMPOSABLE INTEGRAL REPRESENTATIONS
}

\author{
BY A. HELLER AND I. REINER ${ }^{1}$ \\ Communicated by Daniel Zelinsky, January 19, 1962
}

1. Introduction. The purpose of this note is to sketch a proof of the following theorem.

THEOREM. If $G$ is a finite group having finitely many non-isomorphic indecomposable integral representations then for no prime $p$ does $p^{3}$ divide the order of $G$.

It is known that the same hypothesis implies that all the Sylow subgroups of $G$ are cyclic; thus they are cyclic of order $p$ or $p^{2}$. We do not know whether the converse is true. On the other hand, we have shown elsewhere [1] that a cyclic group of order $p^{2}$ has finitely many non-isomorphic integral representations.

In the same place it is shown that the above theorem follows from this proposition:

Proposition. Let $G$ be a cyclic group of order $p^{3}$. Then $G$ has infinitely many non-isomorphic indecomposable representations over the p-adic integers.

We outline below the proof of this proposition, which will appear in full elsewhere.

2. Construction of indecomposables. Let $\Lambda$ be a ring such that the Krull-Schmidt theorem holds for finitely generated left $\Lambda$-modules; this is certainly the case for algebras of finite rank over a complete valuation ring [3]. We shall write Hom for $\mathrm{Hom}_{\Lambda}$ and Ext for Ext ${ }_{\boldsymbol{A}}^{1}$.

Suppose that $M$ and $N$ are indecomposable $\Lambda$-modules such that $\operatorname{Hom}(M, N)=0, \operatorname{Hom}(N, M)=0$. If $M^{(k)}$ is a direct sum of $k$ copies of $M$ then $\operatorname{Hom}\left(M^{(k)}, M^{(k)}\right)$ may be identified with the ring of $k \times k$ matrices with entries in $H=\operatorname{Hom}(M, M)$. Also $\operatorname{Ext}\left(N^{(u)}, M^{(t)}\right)$ consists of $t \times u$ matrices with entries in $\operatorname{Ext}(N, M)$. If $H^{\prime}=\operatorname{Hom}(N, N)$ then $\operatorname{Ext}(N, M)$ is an $\left(H, H^{\prime}\right)$-bimodule, and $t \times t$ matrices over $H$ and $u \times u$ matrices over $H^{\prime}$ operate in the obvious way on $\operatorname{Ext}\left(N^{(u)}, M^{(t)}\right)$.

We shall say that a matrix $X \in \operatorname{Ext}\left(N^{(u)}, M^{(t)}\right)$ is decomposable if there are invertible matrices $T$ over $H$ and $U$ over $H^{\prime}$ such that

\footnotetext{
1 The research of the second author was supported in part by a research contract with the Office of Naval Research.
} 


$$
T \times U=\left(\begin{array}{ll}
B & 0 \\
0 & D
\end{array}\right),
$$

where, of course, $B$ and $D$ need not be square matrices.

Lemma 1. An extension $E$ of $N^{(u)}$ by $M^{(t)}$ with extension class $X$ is a decomposable module if and only if $X$ is a decomposable matrix.

In order to apply this lemma it is convenient to observe the following consequence.

Corollary. Let $\tilde{H}, \tilde{H}^{\prime}$ be quotient rings of $H, H^{\prime}$. Suppose $V \subset \operatorname{Ext}(N, M)$ is an $\left(H, H^{\prime}\right)$-submodule and that $\tilde{V}$ is a quotient of $V$ on which $\tilde{H}, \tilde{H}^{\prime}$ operate. If $X$ is a matrix with entries in $V$ whose image $\tilde{X}$ in $\tilde{V}$ is $\left(\tilde{H}, \tilde{H}^{\prime}\right)$-indecomposable then the extension corresponding to $X$ is an indecomposable module.

3. Construction of the submodule. In this paragraph we set $\Lambda=E_{2}$ $=Z_{p}^{*} G_{p}{ }^{2}$, where $Z_{p}^{*}$ is the ring of $p$-adic integers, and $G_{p}{ }^{2}$ is cyclic of order $p^{2}$ with generator $g$. We write $C=\left(g^{p}-1\right) E_{2}$ and $E_{1}=E_{2} / C$. For any module $N$, we shall set $\bar{N}=N / p N$.

Now Ext $\left(C, E_{1}\right) \approx \bar{E}_{1} \approx \bar{Z}[g] /(g-1)^{p}$. We define $M$ to be the extension of $C$ by $E_{1}$ with extension class $g-1$. Since $\operatorname{Hom}\left(E_{1}, C\right)=0$, $\operatorname{Hom}\left(C, E_{1}\right)=0$, we may apply Lemma 1 with $k=1$. Thus $M$ is indecomposable. Further, if $H=\operatorname{Hom}(M, M)$, there is a canonical monomorphism $\rho: H \rightarrow \operatorname{Hom}(C, C)+\operatorname{Hom}\left(E_{1}, E_{1}\right)$ whose image may be described as follows [2].

LEMMA 2. $\rho(H)$ consists of pairs $\left(a_{L}, b_{L}\right)$, where $a, b \in E_{2}$ and the subscript $L$ denotes left multiplication, such that

$$
(g-1)(a-b) \in p E_{2}+(g-1)^{p} E_{2} .
$$

Denoting by $\operatorname{rad} H$ the Jacobson radical of $H$, we have the following consequence.

Corollary. $\rho(\operatorname{rad} H)$ consists of pairs $\left(a_{L}, b_{L}\right) \in \rho(H)$ such that $a, b \in \operatorname{rad} E_{2}=p E_{2}+(g-1) E_{2}$. Thus $\tilde{H}=H / \operatorname{rad} H \approx \bar{Z}$.

Although $M$ is indecomposable this is not true of $\bar{M}$. We have instead the following result.

LemMA 3. $\bar{M}=E_{2} u \oplus E_{2} v$ as an $E_{2}$ module, where $p u=p v=(g-1) u$ $=(g-1)^{p^{2}-1} v=0$.

Now let $V$ be the submodule $E_{2} u+E_{2}(g-1) v$ of $\bar{M}$. Then, as a consequence of Lemma 2 , we have the following result. 
Lemma 4. $V$ is an $H$-submodule of $\bar{M}$ and $(\operatorname{rad} H) V=E_{2}(g-1)^{2} v$. Thus $\tilde{V}=V /(\operatorname{rad} H) V$ is a two-dimensional $\tilde{H}$-space with basis $\tilde{u}, \tilde{v}$, the images of $u$ and $(g-1) v$.

4. Proof of the proposition. We now change our notation so that $\Lambda=E_{3}=Z_{p}^{*} G_{p}{ }^{2}$ where $G_{p}{ }^{3}$ is cyclic of order $p^{3}$ with generator $g_{3}$. Then $g_{3} \rightarrow g$ defines a ring epimorphism $E_{3} \rightarrow E_{2}$; we use this to turn all $E_{2}$ modules into $E_{3}$-modules.

If $N=\left(g_{3}^{p^{2}}-1\right) E_{3}$, and $M$ is the module defined in $\S 3$, then $\operatorname{Hom}(M, N)=\operatorname{Hom}(N, M)=0$ and $\operatorname{Ext}(N, M) \approx \bar{M}$. But $H^{\prime}$ $=\operatorname{Hom}(N, N)$ consists only of left multiplications $a_{L}, a \in E_{3}$. Thus $\left(\operatorname{rad} H^{\prime}\right) V=E_{2}(g-1)^{2} v$ and $\tilde{H}^{\prime}=H^{\prime} / \operatorname{rad} H^{\prime} \approx \bar{Z}$ operates on $\tilde{V}$.

We are now in a position to apply the corollary to Lemma 1 . For any integer $k$ let $X^{(k)} \in \operatorname{Ext}\left(N^{(k)}, M^{(k)}\right)$ be the matrix $X^{(k)}=u I$ $+(g-1) v J$, where $J$ is any $k \times k$ indecomposable matrix over $\bar{Z}$. Since the matrices $\tilde{X}^{(k)}=\tilde{u} I+\tilde{v} J$ are clearly $\bar{Z}$-indecomposable, i.e., $\left(\widetilde{H}, \widetilde{H}^{\prime}\right)$-indecomposable, the same must be true of the corresponding extensions.

\section{REFERENCES}

1. A. Heller and I. Reiner, Representations of cyclic groups in rings of integers. I, Ann. of Math. (to appear).

2. A. Heller, Homological algebra in abelian categories, Ann. of Math. 68 (1958), 484-525.

3. R. G. Swan, Induced representations and projective modules, Ann. of Math. 71 (1960), 552-578.

UNIVERSITY OF ILLINOIS 